\title{
Hematological changes after Röntgenray exposure in radiologic technologists
}

\begin{tabular}{rrrr}
\hline Govand Tawfeeq $^{1}$ & Rawaz Tawfeeq $^{1 *}$ & Aram Ommar $^{2}$ & Shakar Ali $^{2}$ \\
\hline Abstract
\end{tabular}

Background and objective: In view of the known health hazards of X-ray radiation, this study focuses on the outlined effect of prolonged accidental or work-related Röntgenray exposure to hematological parameters such as red blood cells (RBCs), white blood cells (WBCs), platelets count as well as serum malondialdehyde (MDA) and glutathione in X-ray technicians.

Methods: In this cross-sectional study with a comparison group, blood samples were collected over six months period from X-ray technician assistants across Rizgary teaching hospital, maternity teaching hospital, Nanakaly hospital, Erbil teaching hospital, Ashty hospital, Hundreen hospital, and Harem hospital. A convenience sample was chosen for collecting subjects exposed to X-ray at the radiology department in the hospitals mentioned above. The results were analyzed and compared with a second control group of healthy unexposed individuals.

Results: It was determined that prolonged Röntgenray exposure could lead to an increase in the amount of oxidative stress as denoted by the decrease in the levels of reduced glutathione in the plasma of our test subjects.

Conclusion: Although there were changes in the mean levels of plasma RBC, WBC, platelets, and malondialdehyde, the differences of these parameters between the two groups were not significant statistically. However, there was a significant reduction in glutathione levels in plasma samples of the subjects, indicating elevated oxidative stress levels within the body.

Keywords: X-ray; Blood cells; Malondialdehyde; Glutathione.

\section{Introduction}

X-rays are part of the electromagnetic spectrum consisting of electric and magnetic fields traveling perpendicularly. This spectrum also includes radio waves, microwaves, and visible light. Ionizing radiation includes alpha, beta, and gamma rays and neutrons with sufficient energy to generate ion pairs, i.e., electrons. These electrons can generate chemically active free radicals, which can damage the molecular structure leading to cell dysfunction (somatic effect) or mutations (genetic damage) ${ }^{1}$ Ionizing radiation at a higher intensity, meaning least or no protected barrier for those working with
X-rays, than those used in radiological practices can penetrate living tissues and destroy living cells or make them abnormal in function. ${ }^{2}$ Workers exposed to X-ray radiation are prone to develop lifethreatening diseases often related to the hematopoietic system. The hematopoietic system is highly sensitive to radiation, and therefore the peripheral blood count may serve as a biological indicator of such damage. ${ }^{3}$ Blood cell count studies have been held that addressed the effects of partial or total body irradiation on peripheral blood cell count. ${ }^{4,5}$ The metabolic effect on the hematopoietic system is based on the magnitude and

${ }^{1}$ Department of Clinical Analysis, College of Pharmacy, Hawler Medical University, Erbil, I raq

2 Department of Pharmacology, College of Pharmacy, Hawler Medical University, Erbil, I raq.

* Correspondence: rawaz_dlzar@yahoo.com 
duration of the exposure. The amount of radiation at which it can be considered to be a lethal dose of full body exposure to ionizing radiation is called the "mean fatal dose" and amounts to about 500-600 Rems. ${ }^{6}$ It has been demonstrated in multiple studies that changes in hematological parameters such as those of RBCs, WBCs, and platelets occur when blood is exposed to high doses of X-rays. ${ }^{7}$ As mentioned beforehand, these hematological parameters can be used to evaluate approximate damage to the hematopoietic system. By comparing them with levels found in healthy individuals, we can warn individuals to take more suitable levels of precaution. We may also evaluate the severity of prolonged over-exposure to Röntgen ray. Glutathione is a natural antioxidant present within the cells comprising three key amino acids: Cysteine, glutamate, and glycine. Furthermore, glutathione detoxifies certain chemicals and plays a vital role in neutralizing harmful free radicals. A deficiency of glutathione is often an indicator of the presence of oxidative stress. ${ }^{8}$ Malondialdehyde (MDA) is notably well known for its role as a marker for oxidative stress. Hence, it has been suggested that individuals who are exposed to unusually high levels of radiation may have higher levels of MDA. ${ }^{9}$ The present study aimed to demonstrate the impact of prolonged $X$-ray exposure on serum oxidative markers and the complete blood count. It also aimed to assess the severity of chronic exposure to X-rays and compare the results with a control group, discuss the factors contributing to changes in serum MDA or glutathione, and construct recommendations for prophylaxis of radiation poisoning.

\section{Methods}

This cross-sectional study with a comparison group was conducted over six months within the College of Pharmacy in Hawler Medical University during the year 2015. In our study, we performed the experiment twice to ensure repeatability and accuracy. Forty-two subjects participated in this study from many different hospitals such as Rizgary teaching hospital, Maternity teaching hospital, Nanakaly hospital, Erbil teaching hospital, Ashty hospital, Hundreen hospital, and Harem hospital in the city of Soran. The duration of exposure for these workers was between 1 to 22 years. Most of these X-ray technicians worked in different shifts; however, most technicians worked for five days per week in shifts lasting up to 6 hours per day. A minority of them worked for three days a week. They were age matched and gender-matched with a second group containing healthy (un-exposed) subjects. These included individuals from various backgrounds and professions as inclusion criteria. However, exclusion criteria were indicated that any healthy subjects consuming any medicine, which some of them affect hematological parameters, and taking the iron supplement were excluded. Moreover, any participants suffering from diseases like diabetes mellitus, cardiovascular disease, and all these diseases making alteration in the oxidative stress biomarkers and antioxidants system in both groups were excluded. In this study, we selected two volunteer groups; the first group consisted of 21X-ray technician assistants. The second group was a control group consisting of 21 healthy individuals who were not exposed to Röntgen rays frequently. In both groups, approximately half of the subjects were males while the others were females. Technician assistants between the ages of 24 to 52 years were selected to be part of the first group from the Radiology Departments in different hospitals located in Kurdistan Region, north of Iraq. The subjects are selected as a convenience sample at the first stage while being randomly at the second stage for selecting the control group. There are a population dealing with X-ray at Radiology department in all hospitals and samples were chosen among this 
population in all hospitals with their consent to participate this study. In terms of antioxidant parameters such as MDA levels as well as reduced glutathione. In the second part of this study, 16 subjects participated within the $\mathrm{X}$-ray exposure group, with 16 healthy subjects from the healthy group mentioned. These two groups (each composed of 16 subjects) undergone antioxidant measurements are the same as those that participated with hematological parameters in both groups. Two milliliters of blood were collected from each subject by venipuncture and a disposable syringe. The blood samples were subsequently transferred to a test tube containing the anti-coagulant Ethylene diamine tetraacetic acid (EDTA) in a concentration of $1.5 \mathrm{mg} / \mathrm{ml}$. These collected blood samples were used for blood cells count. Each specimen was labeled with the subject identification code number. The Blood cell count was calculated using an electronic cell counter (Beckman coulter counter, model KX-21N Sysmex). In our second part of this study, sixteen blood samples from each X-ray exposure and control groups were consumed for antioxidant measurements. In addition, the serum MDA and glutathione as antioxidants were compared between the control group and the subject group. To separate glutathione and MDA, blood samples were taken and left to clot for 20-30 minutes, followed by centrifugation of the samples and separation of the plasma compartment in fresh test tubes. The plasma samples were stored in $-20{ }^{\circ} \mathrm{C}$ and used within 1-2 months. The level of each of MDA and glutathione were measured using ELISA technique (Human MDA ELISA kits, Catalog number: MBS728071 with a sensitivity of $1.0 \mathrm{ng} / \mathrm{ml}$ and Human glutathione ELISA kits, Catalog number: MBS042904 with a sensitivity of $1.0 \mu \mathrm{mol} / \mathrm{L}$ ).

\section{Statistical Analysis:}

The results were computed using the Excel program. The statistical analysis was conducted using two independent samples t-test, and the level of significance was taken as $P \leq 0.05$.

\section{Results}

The hematological parameters for RBC, WBC, and platelet count in X-ray technicians for both experimental groups are presented in Table 1 and Table 2, respectively. There are no significant differences in RBCs and WBCs parameters between the two groups while noticing some numerical discrepancies between their numbers. Nonetheless, platelet measurements are considerably higher in the X-ray exposure group than the control one, as shown in Table 1.

Table 1: Means \pm SD of the hematological parameters of the exposed and unexposed groups.

\begin{tabular}{lccc}
\hline Parameters & Control $(\mathrm{N}=\mathbf{2 1})$ & $\begin{array}{c}\text { X-Ray exposure } \\
(\mathrm{N}=\mathbf{2 1})\end{array}$ & $\boldsymbol{P}$ value \\
\hline RBCs $\left(10^{*} / \mu \mathrm{l}\right)$ & $5.34 \pm 0.80$ & $5.71 \pm 0.6$ & 0.070 \\
WBCs $\left(10^{* 3} / \mu \mathrm{l}\right)$ & $5.95 \pm 0.74$ & $6.88 \pm 0.55$ & 0.060 \\
Platelets $\left(10^{* 3} / \mu \mathrm{l}\right)$ & $255.11 \pm 15.63$ & $295 \pm 11.35$ & 0.049 \\
\hline
\end{tabular}


Table 2 shows no statistically significant differences between RBC, WBC, and platelet levels in 21 test subjects compared to the baseline levels in the sample group of X-ray exposure only. In our next set of data, serum MDA levels and reduced glutathione in medical workers were compared with normal serum levels in the control group, as illustrated in Table 3 . A statistically significant difference was only found in glutathione levels in the 16 test subjects compared to the control group being 16 , as can be demonstrated by the obtained $P$ value.

\section{Discussion}

The objective of the current study was to examine hematological parameters in technicians working in hospitals due to the apparent effect of radiation on these parameters. In our study, there are no significant differences in levels of RBC or WBC. This could be because X-ray technicians were exposed to low doses of radiation since the highest protection levels are applied in hospital practice. For example, in a similar study conducted by Taqi et al. (2018), there were no statistically significant changes to WBC, hemoglobin, and platelets between X-ray technicians working between 1 to 6 hours per day and control subjects. Interestingly the study discovered a significant increase in RBC for X-ray technicians. ${ }^{10}$ This could be illustrated by studies that indicate high radiation levels resulted in a significant reduction of individuals' blood count levels. High levels of radiation mean working without any protective barriers or exposure for long times at radiological departments. Zachariah et al. (2001) assessed weekly blood count levels to identify a pattern of change in blood cell count during conventional radiotherapy. ${ }^{11}$ The study revealed a statistically significant reduction in all blood cell counts. Although in the present study, the reduction was not deemed clinically significant, previous studies have established that radiation can produce irreversible changes in the DNA of white blood cells. A link between an increase in oxidative stress and the formation of cancer has been established. Despite controversy about the effects of $X$-rays on hematological parameters, many studies prove the same results as ours. In a paper published by Wagiallah (2013), it was reported that workers handling X-ray equipment had disturbances in leucocytes, neutrophils, and lymphocyte

Table 2: The second round of experiments held to determine the effect of long-term X-ray exposure on blood cells count (RBC, WBC, and platelets) in 21 test subjects.

\begin{tabular}{lcc}
\hline Parameters & Irradiated group & $\boldsymbol{P}$ value \\
\hline RBCs $\left(10^{*} / \mu \mathrm{l}\right)$ & $5.78 \pm 0.19$ & 0.710 \\
WBCs $\left(10^{* 3} / \mu \mathrm{l}\right)$ & $6.75 \pm 0.23$ & 0.590 \\
Platelets $\left(10^{* 3} / \mu \mathrm{l}\right)$ & $234.02 \pm 8.97$ & 0.720 \\
\hline
\end{tabular}

Table 3: The measurement of malondialdehyde and glutathione in X-ray technicians and control groups.

\begin{tabular}{lccc}
\hline Parameters & Control (16) & X-Ray exposure (16) & Statistical decision \\
\hline Malondialdehyde $\mathrm{ng} / \mathrm{ml}$ & $1.5 \pm 0.114$ & $1.268 \pm 0.039$ & 0.072 \\
Glutathione $\mu \mathrm{mol} / \mathrm{L}$ & $8.582 \pm 0.075$ & $5.394 \pm 0.95$ & $<0.001$ \\
\hline
\end{tabular}


count. However, no statistically significant differences were observed in the number of red blood cells and hemoglobin. ${ }^{6}$ Rozgaj et al. (2002) reported that long-term exposure to low doses of ionizing radiation might affect the cells and tissues and result in various adverse health effects. ${ }^{12}$ Furthermore, it was also reported that the blood count dropped soon after irradiation and recovered within several weeks. In acute exposure, X-ray irradiation has a destructive action on cells of the immune system and depresses their functional activity. In addition, antibody-producing ability, delayed type hypersensitivity reaction, and mutagenic activity were also found to be affected to some degree by X-ray irradiation. As it has been mentioned before, MDA is a reliable biomarker that indicates the relative oxidative stress that an organism is exposed to. Medical radiologists are exposed to low doses over a relatively longer period of time. Thus, some research papers have concluded that exposure to high doses of ionizing radiation can contribute to increases in MDA. However, our work reports no observable changes in blood MDA levels in medical radiologists exposed intermittently to low doses of Röntgen radiation. Studying the data that we have obtained, differences in malondialdehyde level between the control and the group exposed to X-rays was more likely due to other external factors. Since the likelihood of the null hypothesis being rejected is marginal $(P=0.072)$ and bordering on the results being acceptable. Medical staffs at radiological departments in our population have the most protection materials for such deleterious effect of X-ray at hospitals, and so they are less likely to be susceptible to a high dose of X-ray. This is due to a negligent amount of free radical damage caused by membrane lipid peroxidation. This claim is further supported by a research paper published by Navratil et al. (2008) in which four separate groups (blood obtained from volunteers, a nuclear power plant employee, radiotherapy patients, and a control group) were exposed to Rontgen rays. No strong difference was found in MDA levels in the test subjects and the control group. However, it is worth mentioning that differences in glutathione were measured in this experiment. ${ }^{13}$ The dose of radiation applied to a subject is a variable that has a major impact on MDA levels. Intake of MDA may also affect levels when taken in the form of supplementation. ${ }^{15}$ Therefore, it is necessary to minimize any variables which may affect its levels when conducting a study. Examples of variables would include diabetes, cancer, chemotherapy, or cardiovascular disease. In our study, it was also shown that there was a significant decrease in glutathione levels $(P<0.05)$ in medical radiologists who were intermittently exposed to low doses of X-rays. As has been mentioned before, glutathione exists in both the reduced and oxidized state. In the reduced state, the thiol group is capable of donating an electron or conjoining with the oxyradical. It has been clearly documented that a correlation between factors such as oxidative stress, radiation, poor diet, the buildup of free radicals, and the depletion of glutathione exists. ${ }^{16}$ Higher levels of radiation lead to a reduction in glutathione levels. This is well thought to be because of the production of reactive oxygen and nitrogen species produced by the short -lived ionizing radiation, which is then neutralized by the reduced glutathione (GSH) producing glutathione in its oxidized (GSSG) state. The depletion of glutathione can cause a cascade of events, including phospholipase-A2 dependent release of arachidonic acid. Arachidonic acid, being a polyunsaturated omega- 6 fatty acid, stimulates thrombocytes to produce malondialdehyde. ${ }^{2}$

\section{Conclusion}

We concluded that long term accidental or work-related exposure to $\mathrm{X}$-rays does not have a notable effect on the WBC, RBC, platelet, or the MDA levels, thereby further 
verifying previous results that were obtained. Thus, it can be postulated from these results that the level of exposure that X-ray technicians experience is not significant enough to affect the hematopoietic levels, or the effect is not notable enough. An interesting note can be drawn from the results regarding the oxidative stress that $X$-rays have on the human body. According to our results, the test subjects had a significant decrease in reduced glutathione levels within the plasma, signifying oxidative stress within the body. Qualified radiographers are expected to be wearing protective clothing during certain procedures. In developing countries, healthcare professionals may not even be wearing the protective gear needed to protect against radiation poisoning. In the future, it would be thought provoking to see experiments being performed where a possible correlation between glutathione (total, oxidized, and reduced, separately) and MDA levels can be drawn. It may also be beneficial to monitor the indicator Gamma glutamyl transferase since up-regulation of GGT occurs in glutathione deficiency in tissue cells.

\section{Competing interests}

The authors declare no competing interests.

\section{References}

1. Masumura K, Kuniya K, Kurobe T, Fukuoka M, Yatagai F, Nohmi T. Heavy-ion-induced mutations in the gpt delta transgenic mouse: Comparison of mutation spectra induced by heavy-ion, X-ray, and gamma-ray radiation. Environ Mol Mutagen 2002; 40(3):207-15.

2.Adhikari SR. Effect and application of ionization radiation (X-ray) in living organism. Himalayan Physics 2013; 3(3):89-92.

3. Williams JP, Brown SL, Georges GE, Hauer-Jensen M, Hill RP, Huser AK, et al. Animal models for medical countermeasures to radiation exposure. Radiat Res 2010; 173(4):557-78.

4. Wirth MD, Sevoyan M, Hofseth L, Shivappa N, Hurley TG, Hébert JR. The Dietary Inflammatory Index is associated with elevated white blood cell counts in the National Health and Nutrition Examination Survey. Brain Behav Immun 2018; 69:296-303.
5. El-Shanshoury H, El-Shanshoury G, Abaza, A. Evaluation of low dose ionizing radiation effect on some blood components in animal model. J Radiat Res \& App Sciences 2016; 9(3):282-93.

6. Waggiallah, $H$. The effect of $X$-ray radiation on hematopoietic tissue among radiology technologists. NJIRM 2013; 4(2):16-20.

7. Faraj K, Mohammed S. Effects of chronic exposure of X-ray on hematological parameters in human blood. Comp Clin Pathol 2017; 27(1):31-6.

8. Mytilineou C, Kramer B, Yabut J. Glutathione depletion and oxidative stress. Parkinsonism Relat Disord 2002; 8(6):385-7.

9. Ahmad I, Temme J, Abdalla M, Zimmerman M. Redox status in workers occupationally exposed to long-term low levels of ionizing radiation: A pilot study. Redox Rep 2016; 21(3):139-45.

10. Taqi A, Faraj K, Zaynal S, Hameed A, Mahmood A. Effects of occupational exposure of X-ray on hematological parameters of diagnostic technicians. Radiation Physics and Chemistry 2018; 147:45-52.

11. Zachariah B, Jacob SS, Gwede C, Cantor A, Patil J, Casey L, et al. Effect offractionated regional external beam radiotherapy on peripheral blood cell count. Int J Radiat Oncol Biol Phys 2001; 50(2):465-72.

12. Rozgaj R, Kasuba V, Sentija K, Prlic I. The frequency of dicentrics and acentrics and the incidence of rogue cells in radiation workers. Mutagenesis 2002; 17(2):135-9.

13. Navratil L, Jaroslav R, Havrankova R, Beranek L, Skalicka Z, Hana S, et al. Changes in selected parameters of the antioxidant system in radiation damage to the organism. J Appl Biomed 2008; 6(4):195-201.

14. Al-Bazi W, Al-Bazi S. Histological and physiological study about effect of chronic X-ray exposure on male rabbit brain. JKU 2014; 12(1):228-34.

15. Deger Y, Dede S, Belge A, Mert N, Kahraman T, Alkan M. Effects of X-ray radiation on lipid peroxidation and antioxidant systems in rabbits treated with antioxidant compounds. Biol Trace Elem Res 2003; 94(2):149-56.

16. Tiwari S. Oxidative stress and antioxidant defense in cells. Global Journal for Research Analysis 2012; 3(8):11-4. 